\title{
Incidencia del reglamento nacional de nivelación y admisión en el acceso a la educación superior en Ecuador
}

\author{
Frank L. Mila, Ximena E. Maldonado y Karla A. Yánez \\ Universidad de Otavalo, Carrera de Derecho, Av. Sarances y Pendoneros, Otavalo, Ecuador. \\ (correo-e: fmila@uotavalo.edu.ec; xmaldonado@uotavalo.edu.ec; ep_kayanez@uotavalo.edu.ec)
}

Recibido Dic. 3, 2019; Aceptado Ene. 30, 2020; Versión final Mar. 24, 2020, Publicado Jun. 2020

\begin{abstract}
Resumen
Este estudio tuvo como objetivo hacer un análisis del acceso a la educación en el ámbito público de las universidades ecuatorianas. Se realizó una investigación con un enfoque cualitativo, de carácter documental. Se emplearón el método dogmático-jurídico como técnica de recolección de información. Se aplicó el análisis documental, que parte del derecho constitucional a la educación, específicamente en relación al acceso a la misma, en igualdad de condiciones y oportunidades. También se incluye la normativa relativa a la temática y doctrina al respecto. El resultado del estudio determinó la vulneración del derecho al acceso de la educación superior en jóvenes bachilleres, en virtud de que el proceso de admisión se rige únicamente por un examen, que no observa otras aptitudes de los estudiantes. Por tal razón, se recomendó implementar otras estrategias en proceso de admisión, en aras de generar mayores oportunidades de acceso a la educación.
\end{abstract}

Palabras clave: derecho; educación; acceso; educación superior; igualdad de oportunidades; reglamento nacional; nivelación; admisión

\section{Impact of the national leveling and admission regulation on access to higher education in Ecuador}

\begin{abstract}
The objective of this study was to assess the access to education in public Ecuadorian universities. A qualitative approach was performed that was documentary in nature. A dogmatic-legal method was employed using a documentary analysis as a technique for collecting information. This includes the constitutional right to education in terms of equal access, conditions, and opportunities. It also includes regulations related to the subject and doctrine. The results showed that there was violation of the right to access higher education in young high school graduates. This is since the admission process is governed by a single entry exam, which does not examine other student skills. For this reason, it is recommended to implement other strategies in the admission process in order to generate greater opportunities to access education.
\end{abstract}

Keywords: education rights; access to higher education; equal opportunities; national regulation; leveling; admission 


\section{INTRODUCCIÓN}

La educación es considerada como un derecho fundamental Scioscioli (2016), ésta, se compone de un núcleo esencial conformado a su vez por otros derechos, tales como la disponibilidad de la educación, el acceso a la educación, la permanencia en el sistema educativo y la calidad de la educación. La educación se orienta hacia el desarrollo del sentido de la dignidad de la personalidad humana, como derecho inherente a su naturaleza, que implica que cada persona tiene derecho a ser capacitada y formada, en aras de participar en una sociedad libre, que favorezca la integración de todos los grupos que hacen vida en el Estado. Ahora bien, en el contexto internacional la Declaración Universal de Derechos Humanos expresa que "Toda persona tiene derecho a la educación, la educación debe ser gratuita, al menos en lo concerniente a la instrucción elemental y fundamental", por ello, la educación goza de un reconocimiento universal, el cual abarca su obligatoriedad y gratuidad.

La educación es un derecho humano, de ahí la relevancia del acceso al sistema educativo como medio oportuno para lograr la superación personal, asimismo, se encuentra plenamente reconocido en la Constitución de la República del Ecuador (2008) como parte de los derechos que constituyen el Buen Vivir. El derecho a la educación facilita el acceso al conocimiento, la investigación, la ciencia, la tecnología que permite al individuo un mejor desenvolvimiento como profesional y en los diferentes ámbitos de la vida, (Casanova, 2011). Este derecho, implica obligaciones correlativas que recaen en los actores sociales, tales como la familia, la sociedad y el Estado, cuyo encargo es el goce del derecho a la educación, al igual que brindar una educación de calidad, y en ese sentido, garantizar el acceso al sistema educativo en todos sus niveles o modalidades que buscan promover la formación integral de la persona sustentada en valores tales como: corresponsabilidad, sentido de pertenencia, responsabilidad, el respeto, promoción de la libertad personal y el fomento de los derechos humanos (Latapí, 2009).

En el caso de Ecuador, se cuenta con el Reglamento Nacional de Nivelación y Admisión (2015), instrumento legal que tiene por objeto establecer el proceso de admisión y asignación de cupos universitarios públicos, para garantizar el acceso universal en igualdad de condiciones y oportunidades, en otras palabras, este reglamento norma el proceso de admisión, sin embargo, al momento de su aplicación los aspirantes a un cupo universitario son evaluados únicamente a través de un examen, que mide su capacidad intelectual; y de acuerdo al resultado que obtenga se asigna automáticamente una carrera y universidad, lo cual genera distintas problemáticas para el estudiante como que la carrera asignada no sea acorde a su vocación o destrezas, así como también la movilidad, puesto que se le asigna un cupo en cualquier lugar del país sin tener en cuenta su lugar de residencia.

En el Ecuador, la educación es un derecho con carácter prestacional, que pesar de tener características de exigibilidad, la problemática radica en su ejecución, no depende del ser humano sino de una política estatal y del presupuesto asignado para su realización como política pública. En tal sentido, se plantea como problema científico el siguiente: ¿Cómo incide del reglamento nacional de nivelación y de admisión en el acceso a la educación superior pública en el Ecuador durante el año 2017? Obteniendo como conclusión del análisis realizado que incide en el derecho a la educación en su componente de acceso, puesto que el examen de admisión resulta insuficiente, y, aunque representa una igualdad formal, genera desigualdad material en condiciones y oportunidades. Además, este tipo de sistema genera como consecuencia cada año se incrementa la cantidad de bachilleres sin cupo universitario, dificultándose cada vez más el acceso a la educación superior pública.

\section{EDUCACIÓN SUPERIOR EN EL ECUADOR}

La educación superior resulta un tema de importancia en la actualidad a nivel internacional, en ese sentido, de igual manera es una realidad que presenta algunas dificultades que le circundan, en ese sentido, se aborda en las siguientes secciones el derecho de acceso y permanencia, así como el derecho de libertad en la educación, en el sentido de la escogencia libre, de acuerdo a las capacidades, destrezas y afinidades que posea el futuro estudiante universitario, ello en igualdad de oportunidades, en el marco de la educación superior como un derecho humano universal y del buen vivir. Asimismo, para el caso ecuatoriano es necesario revisar la Ley Orgánica de Educación Superior (2010), el Reglamento de la Ley Orgánica de Educación Superior, y se finaliza, puntualizando sobre los titulares del derecho a la educación y su exigibilidad.

\section{Vulneración del derecho de permanencia a la educación}

La permanencia educativa, es un problema que abarca no sólo al joven sino también a la institución a la cual ingresa, por tal razón, el Estado debe tomar en consideración la escasa formación de los jóvenes al ingresar a las universidades, así como las diferencias propias de cada estudiante que posee características particulares (Carabajal, 2014), para el establecimiento de políticas de permanencia. Ahora bien, la vulneración del derecho de permanencia a la Educación se ocasiona cuando la política pública de educación del Estado 
no brinda seguridad de permanencia a los estudiantes que acceden a un cupo en una IES pública, (Cisternas, 2017), debe tenerse en cuenta que el artículo 26 de la Constitución de Ecuador se refiere al derecho a la educación como un derecho de las personas a lo largo de su vida y un deber ineludible e inexcusable del Estado. Ante esta idea la Secretaria de Educación Superior, Ciencia, Tecnología e Innovación de la Educación Superior del Ecuador (SENESCYT), como ente rector en la materia, debe tener como propósito ampliar la oferta de cupos para que los bachilleres puedan acceder a una educación superior gratuita y de calidad. Asimismo, debe promover en los aspirantes el desarrollo de habilidades y destrezas necesarias para su desempeño profesional acorde a su vocación y decisión, con la finalidad de disminuir la necesidad de acudir a una institución de educación privada, que obliga a financiar sus estudios.

En vista de lo anterior, la no gratuidad de la educación conduce las políticas públicas educativas al terreno de la cofinanciación, lo cual, desde el punto de vista de la garantía del derecho a la educación, independiente de la capacidad de pago, desdibuja el carácter de las obligaciones del Estado de educación obligatoria y gratuita. Sin embargo, la calidad académica que pretende alcanzar la permanencia de los estudiantes en una institución, se ve limitada por la política pública de Estado que aplica un sistema de evaluación que en los últimos años constantemente ha sido reformado, ocasionando inestabilidad, inseguridad jurídica y un estándar no definido de calidad académica, que atenta contra el derecho constitucional a la educación, en su contenido relativo a la permanencia.

El derecho de permanencia va de la mano con la calidad educativa, puesto que este último facilita alcanzar objetivos tanto personales como profesionales. Por otra parte, el Estado debe determinar una política pública de calidad educativa, que tenga como objetivo la permanencia de los estudiantes, para ello, incluso existen diversas estrategias, tales como la propuesta por Burke (2009), quien hace referencia a la retroalimentación de los estudiantes que pueden aportar a la educación superior, explorando sus percepciones, lo cual permite identificar estrategias basadas en las posiciones iniciales de los estudiantes, lo cual incidirá en el desarrollo de su carrera.

\section{Vulneración del derecho de libertad en la educación}

El derecho de libertad referida al acceso a la educación se fundamenta en la libre elección del estudiante para escoger la carrera, así como la institución de preferencia para obtener una profesión a futuro (Cortés, 2012). En el mismo sentido, el derecho de libertad a la elección de universidades, promueve la competencia entre las instituciones de educación superior, lo cual redunda en la calidad de la educación (Prieto y Villamor, 2012). Herrera (2015), señala que la política educativa debe relacionarse profundamente a la planificación económica y social, en la cual deben observarse tres aspectos fundamentales: el primero relacionado con las prioridades y necesidades del país en relación al plan de desarrollo nacional; el segundo, transformando dichas necesidades en objetivos concretos; y el tercero, en la implementación directa en el sistema.

Por su parte, en el Ecuador consagra el derecho a la educación en diferentes planos, incluyendo las políticas públicas que deben ser consideradas prioritarias para garantizar las condiciones necesarias para su pleno reconocimiento y ejercicio. Adicionalmente, esta debe centrarse en el ser humano, lo cual abarca la libertad del mismo, como sujeto acreedor de derechos humanos. Asimismo, la libertad en la educación, se constituye como un principio educativo, para lo cual el Estado debe garantizar la pluralidad de oferta académica, para garantizar el derecho a la libertad de elección. Por otra parte, el Estado ecuatoriano se maneja con una evaluación de conocimiento o examen de admisión, que mide únicamente la capacidad intelectual del aspirante, excluyendo otros aspectos o aptitudes, que de igual manera juegan un papel importante, a la hora de su selección, como, por ejemplo, su estatus socio-económico, su perfil, destrezas, preferencias y demás aptitudes.

\section{La educación superior como un derecho humano universal}

El derecho a la educación superior se sustenta fundamentalmente en la Declaración Universal de Derechos Humanos (DUDH) que señala, que toda persona tiene derecho a la educación gratuita, la instrucción técnica y profesional habrá de ser generalizada: el acceso a los estudios superiores será igual para todos, en función de los méritos respectivos. Por otra parte, el sistema interamericano de derechos humanos reconoce al derecho a la educación en todos sus niveles, así como también su gratuidad, accesibilidad de los estudiantes en igualdad de condiciones y oportunidades. De lo anterior se entiende que la educación superior permanece integrada en la normativa internacional de los Derechos Humanos como parte del derecho a la educación (Jongitud, 2016). Específicamente, el artículo 26 de la DUDH establece que: 1. Toda persona tiene derecho a la educación. La educación debe ser gratuita, al menos en lo concerniente a la instrucción elemental y fundamental. La instrucción elemental será obligatoria. La instrucción técnica y profesional habrá de ser generalizada; el acceso a los estudios superiores será igual para todos, en función de los méritos respectivos. 2. La educación tendrá por objeto el pleno desarrollo de la personalidad humana y el fortalecimiento del 
respeto a los derechos humanos y a las libertades fundamentales; favorecerá la comprensión, la tolerancia y la amistad entre todas las naciones y todos los grupos étnicos o religiosos; y promoverá el desarrollo de las actividades de las Naciones Unidas para el mantenimiento de la paz. 3. Los padres tendrán derecho preferente a escoger el tipo de educación que habrá de darse a sus hijos.

El Pacto Internacional de Derechos Económicos, Sociales y Culturales concuerda con la Declaración Universal de Derechos Humanos (Scioscioli, 2016), puesto que, el derecho a la educación es un derecho para todos los seres humanos sin exclusión de su condición social o cultural, este derecho es propio de los seres humanos para su acceso, desarrollo integral y profesional, los Estados que han suscrito los instrumentos internacionales de Derechos Humanos relacionados con la garantía del derecho a la educación, deben tener políticas de admisión que promuevan la igualdad de condiciones, oportunidades que contribuyen a mejorar el sistema de educación y crear nuevos espacios para el desarrollo del conocimiento.

Asimismo, a nivel regional, el derecho a la educación es reconocido en el Sistema Interamericano de Derechos Humanos, a través de la Convención Americana de Derechos Humanos, puesto que incorpora y reconoce la educación como un derecho humano, con características como universalidad, gratuidad y obligatoriedad. En ese sentido, Muñoz (2012) apunta que se deben promover actividades de enseñanza y aprendizaje dirigidos a grupos étnicos, religiosos, pueblos, comunidades y nacionalidades para conseguir una sociedad organizada, participativa y democrática, ya que es un derecho exigible e intrínseco de los seres humanos. La educación en derechos humanos obedece a una dialéctica que reconoce en los individuos y en los pueblos, no solamente sus derechos como sujetos, sino su papel de participación en la construcción de una sociedad democrática que nos llevará a su efectivo goce. En síntesis, la educación es el motor del desarrollo individual y de la sociedad, siendo un bien público y Derecho Humano que abarca a todos. Asimismo, supera la concepción de un simple servicio educativo y trasciende al Estado y personas, por tanto, el primero tiene la obligación de asegurar el mismo y las personas, poseen derechos propios enlazados con la ciudadanía, tales como en el plano de la libertad de expresión, la participación, todo lo cual incide en el desarrollo de la sociedad (Ascolani, et al., 2018).

\section{La educación como derecho del buen vivir e igualdad de oportunidades}

En el Estado ecuatoriano se considera a la educación como uno de los derechos del buen vivir. Los derechos del Buen Vivir son entendidos como. Indican Caria y Domínguez (2016) que "El concepto de buen vivir ha atraído un interés mucho más allá de su origen en la tradición étnica andina. Se está debatiendo internacionalmente como una contribución a la teoría del desarrollo y se ha convertido en el propósito fundamental de la política de Ecuador desde la adopción de la nueva constitución en 2008". El derecho a la educación como derecho del buen vivir es reconocido por la Constitución de la República del Ecuador en su artículo 26, que expresa: La educación es un derecho de las personas a lo largo de su vida y un deber ineludible e inexcusable del Estado. Constituye un área prioritaria de la política pública y de la inversión estatal, garantía de la igualdad e inclusión social y condición indispensable para el buen vivir. Las personas, las familias y la sociedad tienen el derecho y la responsabilidad de participar en el proceso educativo (Constitución de la República del Ecuador, 2008).

Expresa Benito (2017) que "La Constitución de 2008, concedió especial importancia al Buen Vivir, y para ello, dedicó un importante número de artículos a dar directrices a los poderes públicos sobre la obligatoriedad de alinear las políticas públicas sobre la obligatoriedad de alinear las políticas públicas a dicho enfoque, modo y objetivo de vida para la ciudadanía". Como se puede evidenciar, la Constitución de la República del Ecuador guarda armonía con lo señalado en los Instrumentos Internacionales de Derechos Humanos. Ante ello el Estado debe fortalecer políticas económicas, sociales y educativas que aseguren el libre acceso e ingreso a la educación pública en el Ecuador sin distinción alguna. Asimismo, Latapí (2009) al respecto se entiende que el derecho humano a la educación se recoge en las actas constitutivas de la ONU y, particularmente, en la de su organismo especializado en la educación, la ciencia y la cultura "UNESCO", así como en las Constituciones de muchos países; por tanto, es hoy una de las preocupaciones fundamentales del desarrollo mundial.

Por otra parte, en relación a los indígenas, el artículo 14 de la Declaración sobre los Derechos de los Pueblos Indígenas recientemente adoptada por la ONU (13 de septiembre de 2007), expresamente indica que "derecho a establecer y controlar sus sistemas e instituciones docentes, y a que los indígenas, en particular los niños, tengan derecho a todos los niveles y formas de educación del Estado sin discriminación; y que esa educación se imparta en su propia cultura y en su propio idioma (Latapí, 2009). Lo expresado se suma a lo indicado con anterioridad, relacionado con la incidencia que puede generar medir la aptitud de un joven, únicamente considerando aspectos basados en una única evaluación, lo cual se agrava en caso de los indígenas, que pertenecen a una realidad particular que no puede ser medida de la misma manera que para los jóvenes no indígenas. En razón de lo anterior, se debe hacer referencia al principio de igualdad de oportunidades al acceso a la educación superior, el cual se ve limitado en virtud de la discriminación generada por no considerarse aspectos culturales propios del Ecuador, tal como el caso de la justicia indígena, que es reconocida en la Constitución. 
Al respecto, la SENESCYT, como ente rector de la política de admisión y nivelación educativa, no cuenta con instrumentos que fijen parámetros de ingreso a una institución pública de educación superior, considerando aspectos colaterales, como los expresados en este trabajo, incluyendo el idioma propio de las distintas culturas reconocidas en el Ecuador, siendo esto un claro ejemplo de la afectación al derecho a la educación, en perjuicio este grupo de personas, evidenciándose la falta de oportunidades brindadas por el Estado. En consecuencia, en un contexto de este tipo, es necesario postular que, para avanzar en la comprensión de la relación entre igualdad de oportunidades y educación, no alcanza con un enfoque basado exclusivamente en paradigmas científicos o técnicos.

En ese sentido, es importante destacar que el Estatuto Orgánico de Gestión por Procesos de la SENESCYT (2012) en el artículo 8 hace referencia al acceso de los bachilleres aspirantes al ingreso de la educación superior pública, bajo los principios de meritocracia e igualdad de oportunidades, sin embargo dentro del sistema ecuatoriano se han evidenciado limitaciones para obtener un cupo universitario en las universidades públicas del país, en virtud de los aspectos supra indicados, lo cual incide en el artículo 26 de la Constitución que consagra el derecho a la Educación. Por último, partiendo del libre desarrollo de la personalidad, este abarca el derecho de una persona a escoger libremente sus estudios, sin que sea impuesto por ningún organismo de educación.

\section{La ley orgánica de educación superior (2010)}

El Estado Constitucional de Derechos y Justicia Ecuatoriano, promueve un sistema educativo de inclusión y gratuidad (Pérez, 2004), es fundamental que el Estado apoye a los estudiantes en el ingreso a la educación superior, considerando factores como mejores niveles de escolaridad y calidad de vida, por tanto, es importante que las Instituciones de Educación Superior contribuyan a mejorar la atención a la demanda de educación superior, de manera adecuada conforme a las orientaciones de la UNESCO, basados en la selección de méritos y aptitudes de los aspirantes, respetando y aplicando el principio de igualdad de oportunidades garantizados por la Constitución y por el Sistema Internacional de Derechos Humanos, consagrado en la Constitución de la República del Ecuador (CRE, 2008).

Ahora bien, en función de lo anterior, en el año 2010, entró en vigencia la Ley Orgánica de Educación Superior (LOES), la cual se inscribe en una línea que pretende sumarse a la etapa de cambios para la mejora de los indicadores de calidad en las instituciones de educación superior (Ruíz, et al., 2018). Asimismo, la aludida ley descansa en diversos principios que sirven de eje para la educación, tales como la libertad de enseñanza, la libertad de cátedra, interculturalidad, autonomía responsable, cogobierno, igualdad de oportunidades, calidad, pertinencia, integralidad y autodeterminación. Adicionalmente, se estatuye que a educación debe ser humanista, considerado como un bien público de carácter social, que responde al interés público, tal como se desprende de los artículos 29 de la Constitución y 52 del aludido instrumento legal.

Por otra parte, se puede observar que entre los principios que consagra la ley, figura el de la igualdad de oportunidades, aspecto que se colige con el acceso a la educación, el cual debe sustentarse en una igualdad real, no en apariencia, por ello, existe el fundamento legal para que se dé cumplimiento con tal postulado. En vista de lo anterior, como corolario, el artículo 350 de la Constitución, garantiza la igualdad de oportunidades en el acceso, permanencia, movilidad y en el egreso. En tal sentido, la Constitución genera los esbozos macro relativos a la igualdad de oportunidades en el ingreso a la educación superior en el sector público, así como los principios por los cuales debe regirse el sistema educativo, asimismo, la Ley Orgánica de Educación Superior, desarrolla tales postulados, particularmente en su artículo 81, que dispone la creación un sistema de nivelación y admisión, que responda a los méritos, igualdad de oportunidades y libertad de elección de carrera e institución.

Además, el sistema debe considerar medidas de acción afirmativa para promover la igualdad, en favor de los titulares que se encuentren en condición de desigualdad o vulnerabilidad. Asimismo, el sistema debe considerar las capacidades y competencias de los postulantes, así como su situación o condición socioeconómica, antecedentes académicos, entre otros aspectos, en aras que los mejores puntajes garanticen su ingreso, sin menoscabar, el derecho a otros postulantes que, a pesar de no alcanzar tales puntajes, queden totalmente excluido. Incluso, se consagra que para aquellos casos en los cuales no logren ingresar, se debe asegurar un sistema de nivelación general, con la finalidad de mejorar las capacidades y competencias de los jóvenes, en miras a su posterior ingreso.

Reglamento de la ley orgánica de educación superior (2010)

El Reglamento de Ley Orgánica de Educación Superior (2010) en el capítulo II, consagra el principio de igualdad de oportunidades, considerando que se debe implementar el Sistema de Nivelación y Admisión para el ingreso a las instituciones de Educación Superior pública. Su función principal será la de garantizar el 
cumplimiento de los principios de igualdad, oportunidad, mérito y capacidad, desarrollar la educación superior bajo la perspectiva del bien público social, aportando a la democratización del conocimiento para la garantía de derechos y la reducción de inequidades. Por tanto, este reglamento sive de desarrollo directo de los postulados de la Ley Orgánica de Educación Superior (2010), como norma especial en la materia.

De la misma manera, el Estatuto Orgánico de Gestión por Procesos (SENESCYT, 2012) en el Artículo 8, expresa entre los objetivos estratégicos, que se debe: "Reducir las brechas en el acceso de los bachilleres aspirantes al ingreso de la educación superior, bajo principios de meritocracia e igualdad de oportunidades", dentro del sistema ecuatoriano se ha verificado que existen muchas brechas y limitantes para acceder a un cupo universitario en las universidades públicas del país, sin garantizar lo mencionado en el objetivo estratégico de este estatuto".

Por ello, se debe tomar en cuenta la realidad de los bachilleres, que vienen con una carga y formación previa, que es determinante para su posterior ingreso a la educación superior, tal como afirma Piaget (2010), al aducir que la educación es única y constituye uno de los factores fundamental para la formación intelectual y moral; la escuela es la encargada de orientar al joven estudiante en cuanto a las necesidades e intereses de superación, conducirlo al éxito, la realización de sus propias posibilidades de adaptación a la vida social. En vista de ello, se debe considerar al sistema educativo como uno y no de manera aislada.

Igualmente, existe otro instrumento que sirve de marco normativo, que es el Reglamento del Sistema Nacional de Nivelación y Admisión (RSNNA, 2015), el cual tiene como objeto, según su artículo 1, establecer: "El proceso obligatorio que los aspirantes deben seguir para el ingreso a las instituciones de educación superior públicas, a fin de realizar los estudios correspondientes en los niveles de formación técnica superior, tecnológica superior de tercer nivel, mediante la realización de un examen de habilidades y la superación de las distintas modalidades de los cursos de nivelación y de los distintos instrumentos de evaluación previstos en el Sistema Nacional de Nivelación y Admisión (SNNA)". Como se puede evidenciar, este reglamento tiene por objeto regular el ingreso de los aspirantes a una institución de educación superior pública, a través de la obtención de un cupo universitario, sistema implementado y coordinado por la SENESCYT conjuntamente con el Sistema de Nivelación y Admisión (SNNA); considerando y cumpliendo los principios consagrados en los Instrumentos Internacionales de Derechos Humanos y en la Constitución de la República del Ecuador.

\section{Vulneración del derecho al acceso a la educación}

En el plano internacional, se han generado diversos cambios y transformaciones en los últimos años, motivado a la diversificación y masificación de la educación superior (Burke, 2013), entre los cuales se suma Ecuador, puesto que el acceso a la educación superior cambia a partir de la Constitución del año 2008 y se refleja con mayor énfasis en la Ley de Educación Superior (LOES, 2010), a partir de estas reformas se fundamenta la exigibilidad que tiene el Estado de proveer una educación pública gratuita y de calidad instituyendo ese derecho como un derecho humano, en consecuencia es responsabilidad del Estado y de su política pública que todos los estudiantes que egresen de la educación secundaria tengan un cupo en la educación superior de tercer nivel, garantizando para tal efecto la infraestructura necesaria, por ello, se aduce que se trata de un bien público social y se reafirma en la medida que el acceso a la educación sea un derecho real de todos los ciudadanos (Arizabaleta y Ochoa, 2016).

El derecho a la educación es un derecho social que contiene una garantía que debe ser respetada por el Estado. En el Ecuador este derecho se hace efectivo a través de la ejecución de una política pública que está reglamentada por la Secretaría de Educación Superior Ciencia y Tecnología (SENESCYT) pero que no asegura el derecho social de la educación consagrada en la Constitución y los instrumentos internacionales, estando supeditado a la ejecución de una política pública de derecho-garantía que se convierte de derecho social en derecho civil y por lo tanto exigible no como una garantía sino como una posibilidad, esa posibilidad que viabiliza la calidad académica sin que al parecer se esté vulnerando el derecho, pero realmente se traduce en la evasión de la garantía del derecho a la educación.

Igualmente, el derecho constitucional a la educación es de carácter progresivo, lo cual significa que debe interpretarse y aplicarse de manera que este vaya en mejora y progreso, sin embargo, existen diversos aspectos que pueden afectarlo, tales como la burocracia o la necesidad de legitimar, el poder político con programas que se generan con la premisa de asegurar la calidad de la educación y que pueden erigirse como simples lemas o planteamiento acéfalo, que no son verdaderos mecanismos de acción para hacer efectivos los derechos. Al respecto, la Constitución de la República del Ecuador (2008) reconoce y garantiza los derechos de las personas; siendo de aplicación directa e inmediata, es por eso que el Estado se encuentra obligado a velar por ellos, e intervenir ante cualquier limitación de su ejercicio. Más allá del plano nacional, Según Latapí (2009), el derecho a la educación está consignado en las actas constitutivas de la ONU, siendo evidente la importancia que reviste la educación como un derecho universal, que fundamenta el desarrollo mundial y está considerando como un derecho humano. Asimismo, la UNESCO ha promovido numerosos esfuerzos internacionales tales como el Programa Educación para todos (EPT), entre otros. 
Ahora bien, el Derecho de acceso a la educación, ha sido considerado como parte del núcleo esencial del derecho fundamental a la educación. Esto ha sido desarrollado por la Corte Constitucional de Colombia, mediante decisión № 944, del año 2000, indicó que "Se denomina contenido esencial o núcleo esencial al ámbito necesario e irreductible de conducta que el derecho protege, con independencia de las modalidades que asuma el derecho o las formas en que se manifieste. El núcleo esencial de un derecho fundamental, entonces, no está sometido a la dinámica de coyunturas políticas. En el caso del derecho a la educación, no es posible negar injustificadamente el acceso y la permanencia en el sistema educativo a una persona". (Castellanos, 2018) indica que es por ello, que tiene un contenido esencial que constituye una base de aplicación directa e inmediata, que impide su desconocimiento o desnaturalización, por lo que no es un derecho puramente concebido como una norma programática e insuficiente de protección judicial, pese a la situación de no estar garantizada como otros derechos.

En ese sentido, más allá del plano normativo, es relevante indicar que según Ponce y Carrasco (2017) "la educación y su impacto, en condiciones de equidad socioeconómica, es probablemente la capacidad más importante que puede llegar a tener el ser humano, puesto que genera y potencia otras habilidades personales para acceder a otras necesidades básicas como la alimentación, la salud, el empleo, una vivienda, e inclusive otras condiciones de bienestar como el disfrutar de vacaciones, seguridad, relaciones sociales, entre otros". A esto se debe sumar, que todo parte del acceso a la educación, ya que no se puede entrar a discutir ningún aspecto relacionado con el sistema educativo, si no se aborda en primer término lo atinente a su ingreso.

El derecho a la igualdad al acceso a la educación superior implica que las personas interesadas en la asignación del cupo tienen derecho al mismo, en igualdad de condiciones, a acceder al proceso de selección de los beneficiarios y a que la distribución de los cupos se realice conforme a los procedimientos establecidos. La igualdad de acceso implica que ante la limitación de los cupos, la selección se efectúe siguiendo el criterio del rendimiento académico, con base al principio de igualdad de oportunidades. Igualmente, el acceso al derecho a la educación superior como lo manifiesta Murcia (2007), en el ámbito educativo, para que se reconozca, no debe depender de la capacidad de pago de las familias. Concretamente, la tendencia de las políticas educativas hacia una educación que se puede comprar y vender libremente en el mercado, se traduce en la negación del derecho a la educación, en especial de los grupos poblacionales de menores recursos y de los más vulnerables en su interrelación con el mercado.

El mero reconocimiento del derecho a la educación por parte del Estado, no asegura la efectividad ni goce del mismo, pues hace falta que la política pública que el Estado diseña materialice ese derecho para que en realidad se cumpla con las necesidades de la sociedad. Por otra parte, las instituciones que materializan esa política pública tienen que estar en sintonía con la necesidad de la población, caso contrario se burocratizaría el acceso a la educación superior, se impediría el pleno goce de la garantía reconocida por el Estado y se evidenciaría la característica social de este derecho que es el ser programático. Ahora bien, el Reglamento del Sistema Nacional de Nivelación y Admisión (2015), ciertamente se erige como la norma especializada en la materia, sin embargo, se denota que principalmente toma como aspecto medular del proceso de admisión a la educación superior, el examen nacional para la educación superior (ENES), es decir, se limita a una evaluación, que sólo mide ciertos aspectos intelectuales, lo cual no toma en consideración que existen otros mecanismos que permiten asegurar el ingreso a la educación superior, incluyendo los principios de la educación inclusiva, que es un desafío actual, ya que a medida que los estudiantes con necesidades especiales completan su educación temprana, sienten la necesidad de avanzar hacia prácticas inclusivas en la educación superior (Moriña, 2017).

En vista de lo anterior, es necesario la implementación de un proceso de evaluación para el ingreso, de carácter integral, que no se limite a una evaluación específica escrita, por tanto, debe existir flexibilidad en términos de una población estudiantil diversa (Boelens, et al., 2018). En tal sentido, se realiza un examen idéntico, para un universo de estudiantes que presentan todos características y realidades distintas y diversas, por ello, se debe considerar, entrevistas, actividades extracurriculares, méritos deportivos, antecedentes socio-económicos, de salud, académicos, entre otros, que permitan medir otros potenciales que de igual manera merecen ser valorados. Lo indicado anteriormente, se colige con el contexto internacional que pregona un programa de aptitud académica, en aras de perfeccionar la excelencia y la equidad, tomando en consideraciones la diversidad cultural y socioeconómica, conforme a los derechos humanos, en aras de superar las diferencias y afectación de grupos minoritario, a través de políticas de inclusión, con igualdad de oportunidades, superando así las desventajas educativas y socioculturales que afectan el acceso a la educación, a pesar que los aludidos grupos poseen tales en una alta gama de matices (Mainieri, 2017). Igualmente, aduce la aludida autora, que se puede tomar en cuenta estrategias que partan de la ética, equidad y justicia, para impactar y transformar la sociedad.

Por otra parte, el acceso a la educación se ve afectado por las desigualdades, a nivel internacional. Por ello, existen hallazgos que muestran como el acceso a la educación superior se basa y refuerza en actividades 
sociales, tales como por el pago en secundarias privadas que inflan puntajes, lo cual mejora las posibilidades de acceso, por tanto, es necesario cerrar la brecha entre los menos privilegiados, para lo cual una respuesta es la masificación de la educación, lo cual baja las posibilidades de ingreso de las clase alta, como predilectos constantes al ingreso (Neves, et. al, 2017). Los migrantes latinos, en el caso de Estados Unidos, se enfrentan a mayores desigualdades, por presentar desafíos culturales, lingüísticos y socioeconómicos, ante lo cual se ha evidenciado el aumento del estudio del idioma inglés, así como el aumento de las instituciones de servicio hispano (Flink, 2018), lo cual puede ser replicado en Ecuador, pero, en los casos de jóvenes que manejan otros idiomas correspondientes a cada nacionalidad reconocida, como, por ejemplo, Kichwa.

Entre otro de los aspectos que debe ser tomado en consideración es de los mal denominados indocumentados, ya que constitucionalmente no existe la condición de persona ilegal, en tal sentido, tal como indican (Davidson y Burson, 2017), a pesar que se reconoce tal derecho desde la decisión Plyler v. Doe de 1982, que reconoce dicho derecho a las personas migrantes, la misma debe ser realmente enarbolada, no quedarse en el mero reconocimiento en papel. Igualmente, en el caso de los refugiados, los cuales solo son tomados en cuenta principalmente en los niveles de primaria y secundaria, obviándose la educación superior, lo cual debe ser tomado en consideración, ya que no solo es un derecho, sino una forma de potenciar el desarrollo de un país (Avery, 2018). Otro de los aspectos a considerar, es el relativo al género, ya que se evidencian investigaciones que determinan que existen diferencias importantes en términos de género en el acceso a la educación superior, favoreciendo a los adolescentes, como es el caso de la India o Vietnam, ya que las adolescentes se asocian a tareas domésticas o no cuentan en las aspiraciones de los padres (Sánchez y Singh, 2018).

Por otra parte, el tema de la pobreza es uno de los puntos medulares para la exclusión y la vulneración del acceso a la educación, en virtud de las desigualdades sociales se afecta el rendimiento escolar y la toma de decisión de los estudiantes en los últimos años de las escuelas secundarias (Kosutic, 2017). Por tanto, acceso a la educación se traduce en la superación o prevención de la pobreza, siendo esta última una barrera para el acceso a la educación superior (McNamara, Harvey y Andrewartha, 2019). Ante lo cual, debe tenerse presente esa realidad, ya que, de lo contrario, no se podrá garantizar este derecho a nivel superior, a personas de escasos recursos o situación de pobreza. Igualmente, a nivel europeo, vale destacar que la educación se ha enfrentado a una expectativa adicional que es responder a la demanda, la cual cada vez es más amplia (Jungblut, et al., 2020), para lo cual debe responderse en igual medida, es decir, ampliando la oferta, la cual debe ser cada vez más grande en el Estado ecuatoriano, en aras de disminuir la exclusión.

Por último, en vista de los aspectos indicados anteriormente, se evidencia que el Reglamento objeto de análisis, no se corresponde con el enfoque internacional que se maneja en la temática, ya que el aludido instrumento, se limita a contemplar un proceso, que en realidad está sustentado únicamente en la elaboración de un examen igual para todos los estudiantes, que por razones obvias, presentan particularidades y realidades distintas, lo cual evidencia la vulneración del derecho a la educación, en su apartado referido a la educación superior.

\section{Titulares del derecho a la educación}

Villavicencio (2008) indica lo siguiente: (...) La palabra "derecho" está utilizada en este caso, obviamente, como la facultad o potestad que le asiste a una persona. Y la palabra "humanos" alude a que la única propiedad que ha de satisfacerse para ser titular de estos derechos es la de pertenecer a la clase de los seres humanos. Así, uno de los rasgos más decisivos de los derechos humanos está en su propio nombre, esto es, que el único requisito para ser titular de ellos es ser hombre o mujer, por lo tanto, su posesión no puede estar restringida, bajo ningún respecto, a una subclase determinada de individuos". Al respecto, Latapí (2009) arguye que "las personas interesadas en la educación darán probablemente dos respuestas. Unas contestarán que es el derecho de los individuos a recibir educación según está consignado en el marco jurídico de su país; otros responderán que es un derecho humano que, junto con otros, integra un importante corpus en el derecho internacional; es uno de los derechos económicos, sociales y culturales que han sido proclamados por la Organización de las Naciones Unidas (ONU) desde hace sesenta años como fundamental".

Dicho de otra manera, los derechos humanos son una clase de derechos que se conceden considerando la pertenencia a la especie humana como única propiedad relevante de sus beneficiarios, derivándose de esta idea que los hombres y mujeres poseen un título igual para gozar de tales derechos en la medida en que todos exhiben, en el mismo grado, esa propiedad relevante. (Villavicencio, 2008). Para el caso de los titulares del derecho a la educación de acuerdo a los Instrumentos Internacionales de Derechos Humanos y a la Constitución de la República del Ecuador han definido que la titularidad del derecho a la educación nace del deseo de superación de los seres humanos, destacando que el derecho a la educación es un derecho universal de libre acceso que puede ser ejercido a nivel mundial, a través del Estado, la comunidad y la familia. 
Los especialistas en derechos humanos resaltan la relación del derecho a la educación con los demás derechos humanos. La educación potencia el desarrollo de la persona y por ello es condición esencial para el disfrute de todos ellos; por esto se considera éste como un derecho clave (keyright). No se puede ejercer ninguno de los derechos civiles, políticos, sociales, económicos o culturales sin un mínimo de educación. Por ejemplo: la libertad de expresión: ¿de qué sirve si la persona no tiene las capacidades de formarse un juicio personal y de comunicarlo? O el derecho al trabajo: ¿de qué sirve si se carece de las calificaciones necesarias para un buen trabajo? No sólo la educación es la base del desarrollo del individuo, sino también de una sociedad democrática, tolerante y no discriminatoria; la búsqueda de democracia, de cultura y de paz, la protección del medio ambiente; en suma, la búsqueda del bienestar humano implica que las personas alcancen un nivel mínimo de conocimientos y de capacidades y valores específicamente humanos. (Latapí, 2009). En este contexto los titulares del derecho a la educación son todas las personas naturales, conforme a lo señala en líneas anteriores, en virtud que es un derecho que se reconoce a todo ser humano, basado en el interés que puede tener por la cultura, formación, en atención a sus habilidades.

Con lo manifestado es evidente que la titularidad de los derechos es para todos los seres humanos sin ningún tipo de discriminación en las garantías y oportunidades brindadas por el Estado, por lo que debería efectivizarse tal y como lo reconoce la Constitución de la República del Ecuador, esto es de forma directa e inmediata. Con esta garantía que ha fijado el Estado ecuatoriano el derecho a la educación como política pública debe ser medido, evaluado y mejorado por lo que para cumplir la condición de medición es menester revisar y analizar la política pública de acceso a la educación superior por la aplicación del Reglamento de Nivelación y Admisión. Vivimos en una sociedad de individuos donde las creencias comunes se diluyen y la apuesta a un proyecto colectivo de sociedad se ve cuestionada por un individualismo exacerbado que privilegia los intereses particulares por sobre la preocupación por el bien común.

El Reglamento de Nivelación y Admisión regula al examen nacional de ingreso a la educación superior pública, al que deben someterse los bachilleres para acceder a un cupo universitario, mismo, que está regulado por el Sistema Nacional de Nivelación y Admisión, considerándole a este examen como un instrumento de evaluación de conocimientos por tener un alto impacto personal; puesto que determina el futuro de cada estudiante Registro Oficial Sistema Nacional de Nivelación y Admisión. Al respecto Nogueira (2009) señala: "Sin perjuicio de que se establezca un sistema de becas y un sistema especial de préstamos a quienes no tienen actualmente los recursos para poder cancelar los costos financieros de ella, aun cuando la educación superior no está orientada hacia el objetivo determinado por el Pacto Internacional de Derechos Económicos, Sociales y Culturales de Naciones Unidas que es la implantación progresiva de la gratuidad".

Con lo citado, en el Ecuador no se garantiza el derecho al libre acceso a la educación, así como también se vulnera el principio de gratuidad, por algunos factores, tales como, la movilidad, asignación de un cupo universitario a carreras que no son de vocación del estudiante, entre otras, este sistema que ya ha sido evaluado por parte de los organismos de control y regulación del sistema de educación superior no ha sido la mejor método para poder cubrir la demanda de los bachilleres y con la aplicación de un examen de admisión a través del cual se evalúa la habilidad, capacidad de los aspirantes, aspectos cognitivos tales como aptitud verbal y numérica y el razonamiento abstracto que consiste en ítems que deben resolverse en un tiempo determinado; ocasionando presión y preocupación en los aspirantes, además de que no todos las unidades educativas tienen el mismo nivel de enseñanza con relación a las instituciones públicas y privadas de alto prestigio del Ecuador, y no todos los aspirantes tienen la oportunidad o posibilidad económica para capacitarse por su propia cuenta antes de rendir el examen de admisión, quebrantando así una vez más el acceso universal al derecho a la educación en igualdad de condiciones y oportunidades e incumpliendo con la política pública estatal de gratuidad en la educación. Los nuevos acuerdos multilaterales de comercio internacional colocan a la educación como bien de consumo, como mercancía que se vende y se compra en un mercado mundial. Hoy se discute quien le va a vender educación, a quienes -en el mercado mundial- y bajo qué condiciones podrá hacerlo.

Es necesario señalar que en el Reglamento de Nivelación y Admisión se ha definido el proceso a seguir para que los bachilleres sean parte de este sistema, tales como fijar las fechas de postulación a través de una convocatoria pública nacional que tiene límite de tiempo para su postulación y además se establece la puntuación mínima con la que cada postulante puede aprobar este examen, es importante destacar que para poder ingresar y aprobar el proceso de postulación a las diferentes carreras ofertadas por las instituciones de educación superior es necesario obtener un puntaje de acuerdo a la escala nacional señalada en la convocatoria, caso contrario lo único que les queda a los aspirantes es esperar un nueva convocatoria ya que no pueden acceder a una universidad pública mientras no cuenten con el mínimo requerido además de que está el hecho de que no pueden exceder de dos convocatorias porque el proceso quedaría invalidado.

Finalmente, es importante indicar que el examen de admisión es elaborado por SENESCYT, institución encargada de solicitar y ofertar los cupos que han sido dispuestos por las instituciones de educación superior 
públicas, así como también de los cupos de las instituciones de educación superior cofinanciadas y autofinanciadas conforme la política de cuotas establecido por esta secretaria, lamentablemente estos cupos no son ofertados en relación al número de bachilleres que gradúa el país, sino de acuerdo a la disponibilidad de cada universidad pública, limitando así irremediablemente el ingreso a los postulantes a nivel nacional debido a una falta de espacio e infraestructura de las universidades acreditadas en el país. Por tal razón se puede ver que la política pública de educación superior se encuentra limitada en su aplicación y a pesar de las garantías que determina la Constitución de la República del Ecuador y los Instrumentos Internacionales de Derechos Humanos, pese a ello el Estado ecuatoriano ha evaluado esta situación de acuerdo a los informes presentados por el sistema, para poder controlar y mejorar está política pública y poder hacer efectivo el goce del derecho a la educación en igualdad de condiciones y oportunidades.

\section{La educación como un derecho exigible}

La educación no debe ser concebida o tratada únicamente como una política pública, en virtud que como se ha mencionado en los anteriores capítulos, constituye un Derecho humano y constitucional, que incluso debe ser visto como un sistema, a pesar, que el mismo deba reinventarse y responder a las realidades actuales, al respecto, opina Di Franco (2018), que: "Las universidades han sido hasta aquí el refugio secular de los mediocres, la renta de los ignorantes, la hospitalización segura de los inválidos y-lo que es peor aún- el lugar en donde todas las formas de tiranizar y de insensibilizar hallaron la cátedra que las dictara. Las universidades han llegado a ser así fiel reflejo de estas sociedades decadentes que se empeñan en ofrecer el triste espectáculo de una inmovilidad senil. Por eso es que la ciencia frente a estas casas mudas y cerradas, pasa silenciosa o entra mutilada y grotesca al servicio burocrático. Cuando en un rapto fugaz abre sus puertas a los altos espíritus es para arrepentirse luego y hacerles imposible la vida en su recinto".

Ha existido una constante una pugna entre derechos que son considerados libertades (derechos civiles y políticos) y derechos de carácter prestacional (derechos sociales, económicos y culturales), siendo que la educación suelen inscribirla en estos últimos, cuya prestación va a depender de las capacidades del Estado, sin embargo, por más que se pueda endilgar esta categoría, se trata de derecho universal exigible, que sigue perteneciendo a la familia de los Derechos Humanos. Adicionalmente, en el Estado ecuatoriano no se realiza distinción, por tanto, la justiciabilidad respecto de un derecho significa que puede ser invocado ante los tribunales y ser objeto de tutela por parte del sistema judicial. Igualmente, la exigibilidad de este derecho implica, además de las medidas judiciales, otras de carácter legislativo, político, administrativo o social, conducentes a hacer eficaz un derecho (Latapí, 2009).

El gran debate del derecho durante el desarrollo histórico ha sido definir el ser y el deber ser, en sentido positivo, pues el deber ser es aquello que se espera del derecho y el ser lo que realmente es en la práctica, el derecho desde la perspectiva del ser afinca la posibilidad de realización de sus preceptos y desde el deber ser la forma de exigir esa realización, los actos o comportamientos del Estado marcarían entonces la diferencia entre ese ser y deber ser, en el caso en concreto el ser de la norma constitucional obliga al Estado y lo condiciona a la provisión del derecho al acceso a la educación superior, pero el ser refleja que no se consigue ese derecho debido a los factores que se desarrollan en este estudio.

Por tanto, el derecho de educación es un derecho social que se hace efectivo a través de la ejecución de políticas públicas generadas por el Estado y materializadas a través de la Secretaría de Educación Superior Ciencia y Tecnología (SENESCYT), adicionalmente, tales políticas deben ser armónicas con lo establecido en la Constitución, instrumentos internacionales y normativa legal, que debe vislumbrar una verdadera política ajustada y enfocada al respeto y garantía de tal derecho. La justiciabilidad de los derechos sociales no es asunto sencillo, enfrenta problemas teóricos y prácticos. Entre los primeros se encuentran: la indeterminación conceptual con que suelen estar redactadas las disposiciones constitucionales o la posición de algunos juristas, que consideran que los derechos económicos, sociales y culturales no son verdaderos derechos, sin embargo, la doctrina y normativa internacional, los reconocen de manera unánime. El problema radica en un tema práctico, es decir, su exigibilidad, que va a depender de las capacidades del Estado para responder.

Por otra parte, entre los problemas prácticos se evidencia la inadecuación de los procedimientos jurídicos establecidos para conocer asuntos colectivos (cuando se trata de violaciones a colectividades), pues los mecanismos procesales están diseñados para la defensa de derechos individuales; la falta de jurisprudencia para la interpretación, y otros (Latapí, 2009). En tal sentido, el derecho constitucional de acceso universal a la educación, obliga al Estado a positivizar este derecho, garantizarlo a través de la política pública y no negarlo para así evitar la vulneración al derecho constitucional reconocido en la Constitución de la República del Ecuador del 2008 en su artículo 26. El derecho constitucional a la educación entendido como progresivo, está en riesgo a partir de la creación de la política pública de ejecución por el Estado; debido a varios aspectos, tales como la burocracia o la necesidad de legitimar, el poder político con programas que dicen asegurar la calidad de la educación y que pudieran ser solo membretes y no verdaderos mecanismos de acción para hacer efectivos los derechos. 
Arguye Pérez (2004), que los derechos fundamentales plasmados en la constitución o en un instrumento internacional de derechos humanos, tales como el derecho a la educación no deberían tomarse literalmente, sino sólo en sentido figurado o metafórico, el derecho a la educación supone la obligación de no empeorar la educación. La Constitución de la República del Ecuador reconoce y garantiza los derechos de las personas; puesto que son de aplicación directa e inmediata, es por eso que el Estado se encuentra obligado a velar por ellos e intervenir en aquellos casos, en los cuales cualquier entidad pública, privada o grupo de personas, limiten su libre ejercicio. Por lo que no es extraño enfrentarse con opiniones que, negando todo valor jurídico a los derechos sociales, los caracterizan como meras declaraciones de buenas intenciones, de compromiso político y, en el peor de los casos, de engaño o fraude tranquilizador, sin embargo, algunos autores afirman que incluso los derechos de libertad tienen aspectos relativos al carácter prestacional (Casal, 2008).

En los últimos años, el cuerpo de principios, reglas y estándares que componen el derecho internacional sobre los derechos humanos ha fijado con mayor claridad no sólo las obligaciones negativas del Estado, sino también un cúmulo de obligaciones positivas. Esto significa que ha definido con mayor precisión no solo aquello que el Estado no debe hacer, a fin de evitar violaciones, sino también aquello que debe hacer para lograr la plena materialización de los derechos civiles y políticos y también económicos, sociales y culturales. En tal sentido, los derechos humanos no son pensados hoy tan solo como un límite a la opresión y al autoritarismo, sino también como un programa que puede guiar u orientar las políticas públicas de los Estados y contribuir al fortalecimiento de las instituciones democráticas, particularmente en procesos con democracias deficitarias o débiles (Abramovich, 2006).

Dicho lo anterior, es indudable que el derecho a la educación es reconocido como un derecho fundamental, tiene un contenido esencial que constituye una base de aplicación directa e inmediata, que impide su desconocimiento o desnaturalización. Igualmente, en el plano internacional, el artículo 43 del Pacto Internacional de Derechos Económicos, Sociales y Culturales (1969) expresa: Los Estados Partes tienen obligaciones inmediatas respecto del derecho a la educación, como la garantía del ejercicio de los derechos sin discriminación alguna (párrafo 2 del artículo 2) y la obligación de adoptar medidas (párrafo 1 del artículo 2) para lograr la plena aplicación del artículo 13. Estas medidas han de ser "deliberadas, concretas $y$ orientadas lo más claramente posible" hacia el pleno ejercicio del derecho a la educación.

Cabe reiterar que es incorrecta la concepción de los derechos económicos, sociales y culturales como derechos que establecen exclusivamente obligaciones positivas. Tanto los derechos civiles y políticos como los económicos, sociales y culturales constituyen un complejo de obligaciones positivas y negativas. Las obligaciones negativas son las que tiene el Estado de abstenerse de realizar cierta actividad: no impedir la expresión o difusión de ideas, no violar la correspondencia, no detener arbitrariamente, no impedir que una persona se afilie a un sindicato, no intervenir en caso de huelga, no empeorar el estado de salud de la población, no impedir que una persona acceda a la educación. En cuanto a las obligaciones positivas, conviene establecer algunas distinciones, que nos darán la pauta del tipo de medidas que pueden exigirse. Con cierto automatismo, se suele vincular directamente las obligaciones positivas del Estado con la obligación de disponer de fondos (Abramovich, 2006).

Finalmente, no cabe duda de que esta es una de las formas más características de cumplir con obligaciones de hacer o de dar, en especial en campos como la salud, la educación o el acceso a la vivienda. Sin embargo, las obligaciones positivas no se agotan con el solo hecho de disponer de reservas presupuestarias para ofrecer una prestación. Las obligaciones de proveer servicios pueden caracterizarse por el establecimiento de una relación directa entre el Estado y el beneficiario de la prestación. El Estado puede, sin embargo, asegurar el goce de un derecho a través de otros medios, en los que pueden tomar parte activa otros sujetos obligados (Abramovich, 2006).

\section{DISCUSION}

La realidad mundial actual, impone la necesidad que los futuros profesionales sean formados de forma integral, y con competencias múltiples para enfrentar los retos de la sociedad actual y del futuro, por ello, se deben innovar en el contexto educativo, para que el futuro profesional sea capaz de generar soluciones eficientes y eficaces como autogestor de su profesión, de tal manera que pueda responder con ética profesional, pertinencia y oportunidad a las demandas sociales, que influirán directamente en niveles de vida más satisfactorios (Rodríguez, et al., 2019).

Sin embargo, la aplicación de política pública de educación superior en el Ecuador no es óptima en virtud que el proceso de formulación o estructuración de admisión ha tomado en cuenta que el resultado de este primer momento se convertiría en una política de democratización del acceso a la educación superior, considerando al Examen Nacional de Educación Superior como un instrumento medular para la ejecución de esta política y con un diseño unificado porque es construido por distintos actores seleccionados por el Estado de acuerdo a 
su capacidad y que al finalizar con el diseño de este instrumento se protege su identidad, puesto que en ellos recae la responsabilidad principal para la implementación de esta política, situación que no justifica la consecución de la misma ya que se deja de lado la inclusión y valoración integral de aptitudes para el ingreso al sistema de educación superior, es decir, la política consagrada en el Reglamento Nacional de Nivelación y Admisión de la Educación Superior, realmente no está concebido para que los bachilleres accedan a un cupo universitario público en igualdad de condiciones.

Es importante concluir indicando que una igualdad marginal divide o reparte los medios con igualdad, pero ignora desigualdades relacionadas. Es decir, que mientras que algunos medios necesarios para la competición social son igualados, hay otros que afectan al aprovechamiento de los primeros, que permanecen impunemente desiguales. El derecho a la educación es parte de los derechos sociales, por lo que se considera a este derecho como prestacional; es decir que para su efectivo goce y ejercicio es necesario el cumplimiento de una política pública en manos del Estado, a diferencia de los derechos civiles y políticos que no requieren del Estado para su aplicación directa e inmediata, por tanto, se debe contar con un verdadero sistema de ingreso, aspecto que se determinó en esta investigación, es inexistente, en virtud que se consagra un proceso, empero, gira medularmente en torno a un examen elaborado para toda la población de jóvenes, sin observar sus características, particularidades, diversidad, realidad socioeconómica y otros potenciales que pueden ser traducidos en aptitudes para el acceso al sistema de educación superior.

La equidad dentro de la política de educación superior pública se debe garantizar en todos los niveles, aspecto que no se vislumbra a nivel universitario, en el cual existe una demanda que no se satisface y más allá de eso, en el proceso de selección existe una notoria ventaja por parte de los estudiantes de clases socioeconómicas más altas, ya que se encuentran en mejor situación para afrontar el examen de ingreso. En síntesis, el examen de admisión no es necesariamente el mejor mecanismo para garantizar la equidad en el ingreso, si bien es cierto, que es un instrumento que puede ser utilizado, el mismo debe ser concebido tomando en consideración a todos los actores que intervienen, es decir, albergar la mayoría de la población que es diversa, de ser necesario, contar con diversos exámenes. Igualmente, se determinó que algunas investigaciones coinciden en la existencia de efectos negativos, principalmente para poblaciones con bajo rendimiento en el sistema, favoreciendo así a aquellas cuyo rendimiento es alto, debido a factores estructurales, por lo tanto, la política no ha logrado superar otro tipo de desigualdades asociadas al origen social.

La igualdad de oportunidades no puede limitarse a diseñar un examen igual para todos, sino a la generación de condiciones equitativas dentro de la población objetivo. Al plantear la igualdad de oportunidades de la manera en que se establece en el Sistema Nacional de Nivelación y Admisión y en el examen nacional de ingreso, esta se deriva de una concepción meramente formal, es decir de no discriminación, que va a generar una desigualdad en los resultados, la política de educación superior debe orientarse a una igualdad equitativa de oportunidades, en la cual la población objetivo se encuentre en condiciones "iguales" a través de la asignación de mayores recursos a ciertas poblaciones, es decir que exista desigualdad en la distribución de los mismos.

\section{CONCLUSIONES}

De acuerdo al trabajo realizado y a la discusión presentada, se pueden plantear las siguientes conclusiones principales:

1. La educación se constituye como un derecho humano, establecido en los instrumentos internacionales de derechos humanos a nivel universal y regional. Igualmente, este derecho es un pilar mundial, ya que instituciones como la ONU y la UNESCO, lo promueven de manera categórica. Asimismo, en el caso de Ecuador, se encuentra plenamente reconocido en la Constitución de la República (2008) como parte de los derechos del Buen Vivir, dentro del cual se pregonan derechos de igualdad y no discriminación, y en ese sentido, se ha desarrollado normativa de rango legal, que viabiliza la ejecución de dicho derecho, como lo son la Ley Orgánica de Educación Superior (2010), el Reglamento Nacional de Nivelación y Admisión (2015), el Estatuto Orgánico por Procesos del Consejo de Educación Superior (2012).

2. Se determinó que el derecho a la educación abarca todos los niveles, por lo cual la educación superior es otro de los elementos que debe ser considerado, particularmente, en lo relativo al acceso al sistema de educación superior, en virtud que generalmente los esfuerzos se centran en la permanencia, pertinencia o calidad, obviándose que el punto de partida es el acceso, siendo este uno de los elementos medulares de la educación.

3. Se concluyó que el derecho ecuatoriano contempla una normativa específica que regula la nivelación y el acceso, que consagra un proceso para tal fin, el cual se centra exclusivamente en un examen que es aplicado 
de manera general, sin atender a las distintas realidades socio-económicas, culturales, personales, entre otras, midiéndose a los jóvenes de manera estándar y única, aspecto que incide en el derecho a la educación en su componente de acceso, en virtud que, genera desigualdad y excluye a jóvenes vulnerables del sistema, que de igual manera poseen aptitudes para el ingreso.

4. Adicionalmente, se visualizó que el manejo de un examen para todos los jóvenes incide en el acceso a la educación y afecta principalmente a aquellos que pertenecen a estratos socio-económicos de bajo nivel, a los grupos vulnerables como refugiados, género, migrantes, personas en situación de pobreza, indígenas, y, en general, a personas con necesidades especiales.

5. Entre las recomendaciones para superar tal dificultad, se precisa que se si bien el examen es una herramienta, no puede ser la única, por tanto, debe diversificarse y adicionalmente, se deben implementar otros mecanismos, tales como entrevistas, preparación previa, procurar superar las brechas socioeconómicas, medir otras aptitudes, entre otras.

6. Las anteriores conclusiones se corresponden con la tendencia internacional de diversificar los mecanismos de ingreso a la educación superior, ya que internacionalmente no se maneja un único examen, sino que se busca valorar las aptitudes de los jóvenes que pretenden ingresar al sistema educativo superior.

\section{REFERENCIAS}

Abramovich, V. Una aproximación al enfoque de derechos en las estrategias y políticas de desarrollo. Revista de la CEPAL, 88, 35-50, (2006).

Ascolani, D., Pérez, M.C., Piacentini, J. I. y Piacentini, L. La Educación como Derecho Humano. Comunidad. Revista Digital Lecturas 16 (1), 41-75, (2018).

Avery, H., Learning Practices inside and outside School (LPS), Communication, Culture \& Diversity. Jönköping University, School of Education and Communication, HLK Centre for Middle Eastern Studies at Lund University in Sweden, (2018).

Boelens, R., Voet, M., y De Wever, B. The design of blended learning in response to student diversity in higher education: Instructors' views and use of differentiated instruction in blended learning. Computers \& Education, https://doi.org/10.1016/j.compedu.2018.02.009., 120, 197-212, (2018).

Burke, D. Strategies for Using Feedback from Students in Higher Education, Assessment and Evaluation in Higher Education, https://doi.org/10.1080/0260293080189571134, (1), 41-50, (2009).

Burke, P. J. The right to higher education: Beyond widening participation. Routledge, https://dx.doi.org/10.4324/9780203125571,(2013).

Carabajal, S. La permanencia del estudiante durante el año de ingreso a la Universidad de la República. Una construcción colectiva. InterCambios, 1 (1), 72-81, (2014).

Caria, S. y Domínguez, R. Ecuador's Buen vivir: A New Ideology for Development, Latin American Perspectives https://doi.org/10.1177/0094582X1561112643, (1), 18-33, (2016).

Casanova, M. A. Supervisión y educación inclusiva. Revista de la Asociación de Inspectores de Educación de España, (14), 1-14, (2011).

Castellanos, J. El derecho a la educación como derecho fundamental: Especial atención a su dimensión social prestacional. Revista de educación y derecho, Universidad de Barcelona, (17) 1-12, (2018).

Cisternas, M. S. Derecho a la Educación: Marco jurídico y justiciabilidad. Revista Latinoamericana de Educación Inclusiva. 4 (2), 41-57, (2010).

Constitución de la República del Ecuador, Quito-Ecuador, (2008)

Corte Constitucional de Colombia. Decisión № 944, (2000).

Cortés, F. El derecho a la educación como derecho social fundamental en sus tres dimensiones: educación primaria, secundaria y superior. Estudios Socio-Jurídicos, 14 (2) 185-2015, (2012).

Davidson, T. C. y Burson, K. Keep Those Kids Out: Nativism and Attitudes Toward Access to Public Education for the Children of Undocumented Immigrants. Journal of Latinos and Education, 1 https://doi.org/10.1080/15348431.2016.1179189, 6(1), 41-50, (2017).

Di Franco, M. G. 1918-2018 / 100 de la Reforma Universitaria. Praxis Educativa, https://doi.org/10.19137/praxiseducativa2018-220101, 22 (1), 9-12, (2018).

Flink, P. J. Latinos and higher education: A literature review. Journal of Hispanic Higher Education, https://doi.org/10.1177/153819271770570117, (4), 402-414. (2018).

Herrera, A. Ciencia y política en América Latina, Biblioteca Nacional, Buenos Aires, (2015). 
Jongitud, J. C. El derecho humano a la educación superior en México. Revista de la educación superior, https://doi.org/10.1016/j.resu.2017.02.002, 46(182), 45-56, (2017).

Jungblut, J., Vukasovic, M. y Steinhardt, I. Higher education policy dynamics in turbulent times - access to higher education for refugees in Europe. Studies in Higher Education, https://doi.org/10.1080/03075079.2018.1525697, 45(2), 327-338, (2020).

Košutić, I. The role of cultural capital in higher education access and institutional choice. Center for Educational Policy Studies Journal, 7(1), 149-169. (2017).

Latapí, P. El derecho a la educación, su alcance, exigibilidad y relevancia para la política educativa. Revista mexicana de investigación educativa, 14 (40), 255-287, (2009).

Ley Orgánica de Educación Superior, Quito-Ecuador, (2010).

Mainieri, A. M. Innovations for models of admission Study of cases and inclusion strategies in higher education. Actualidades Investigativas en Educación, http://dx.doi.org/10.15517/aie.v17i3.30215, 17(3), 64-105, (2017).

McNamara, P., Harvey, A., y Andrewartha, L. Passports out of poverty: Raising access to higher education for care leavers in Australia. Children and Youth Services Review, http://doi.org/10.1016/j.childyouth.2017.07.01597, 85-93. (2019).

Moriña, A. Inclusive education in higher education: challenges and opportunities, European Journal of Special Needs Education, https://doi.org/10.1080/08856257.2016.1254964, 32(1), 3-17, (2017).

Muñoz, J. M. La educación inclusiva, una cuestión de derecho. Educatio Siglo XXI, 30(2), 109-128, (2012).

Neves, T., Hélder, F. y Nata, G. Social inequality in access to higher education: grade inflation in private schools and the ineffectiveness of compensatory education. International Studies in Sociology of Education, https://doi.org/10.1080/09620214.2016.1191966, 26(2), 190-210, (2017).

Nogueira, H. Los derechos económicos, sociales y culturales como derechos fundamentales efectivos en el constitucionalismo democrático Latinoamericano. Estudios Constitucionales, http://dx.doi.org/10.4067/S071852002009000200007, 7 (2), 143-205, (2009).

Pacto Internacional de Derechos Económicos, Sociales y Culturales, (1966).

Pérez, A. E. Los derechos Fundamentales. Tecnos, Madrid, (2004).

Piaget, J. Educación en Derechos Humanos. Instituto de investigaciones jurídicas de la UNAM, (2010).

Prieto E., Villamor, M. y Manero, P. Libertad de Elección, Competencia y Calidad: Las Políticas Educativas De La Comunidad de Madrid Profesorado. Revista de Currículum y Formación de Profesorado, Universidad de Granada, 16 (3), 149-166, (2012).

Ponce, J. y Carrasco, F. Acceso y Equidad a La Educación Superior y Posgrado en el Ecuador, Un Enfoque Descriptivo. Mundos Plurales. Revista Latinoamericana de Políticas y Acción Pública, https://doi.org/10.17141/mundosplurales.2.2016.2841, 3 (2), 9-22, (2017).

Rodríguez, L. A., Escobar, M.C., Aveiga, V. I., y Durán, U. C. Estrategia de Formación y Desarrollo de la Competencia Docente llamada Gestionar la Orientación Educativa Familiar, en la Educación Básica Superior. Información tecnológica, http://dx.doi.org/10.4067/S0718-07642019000600277, 30(6), 277-288, (2019).

Sánchez, A., \& Singh, A. Accessing higher education in developing countries: Panel data analysis from India, Peru, and Vietnam. World Development, https://doi.org/10.1016/j.worlddev.2018.04.015, 109, 261-278, (2018).

Scioscioli, S. El derecho a la educación como derecho fundamental y sus alcances en el derecho internacional de los derechos humanos. Journal of supranational policies of education. 2, 6-24, (2016)

Secretaría de Educación Superior, Ciencia, Tecnología e Innovación. Reglamento del Sistema Nacional de Nivelación y Admisión, (2015).

Secretaría de Educación Superior, Ciencia, Tecnología e Innovación. Estatuto Orgánico por Procesos de la Secretaría De Educación Senescyt. Acuerdo № 2011-004. Quito, Ecuador, (2012).

Secretaría de Educación Superior, Ciencia, Tecnología e Innovación. Ficha Informativa de Proyecto 2016. Senescyt. Proyecto: K001 Sistema Nacional De Nivelación Y Admisión. Quito, Ecuador, (2016).

Villavicencio, L. ¿Derechos humanos para quiénes? Reflexiones sobre algunas cuestiones embarazosas. Revista de Derecho Valdivia, http://dx.doi.org/10.4067/S0718-09502008000200002, 21(2), 33-51, (2008). 